\title{
Attitudes to and Understanding of Placebo Use: A Cross- Sectional Exploratory Study in a Malaysian Hospital
}

Amanda Villiers Tuthill, ${ }^{1}$ Tay Zhuo Han, ${ }^{1}$ Chang Xian Chai, ${ }^{2}$ Ai Wen Chai, ${ }^{1}$ Chun Yiing Wong, ${ }^{3}$ Karen Morgan. ${ }^{1,4}$

\begin{abstract}
Background: This study explored doctors' understanding of 'placebo', mechanisms of action, perceptions about effectiveness and concerns about use in a Malaysian teaching hospital. Methods: A survey questionnaire. Results: Respondents were 76 doctors (response rate: $55 \%$ ): $52 \%$ were female, mean age was 32 years, and $61 \%$ were physicians/medical officers. Most (66.2\%) never used a placebo. The main reason for use of placebos was for a possible psychological effect. Placebo use was considered unacceptable due to endangering of doctor-patient trust $(59.2 \%)$ or patient deception $(47.4 \%)$. Conclusion: Developing specific and professional standards and guidance on placebo use could help doctors to leverage the benefits of placebo use without endangering the doctor-patient relationship.
\end{abstract}

Keywords: Placebos; Placebo Effect; Physician-Patient Relations; Health Knowledge, Attitudes, Practice; Malaysia (Source: MeSH-NLM).

About the Author: Tay Zhuo Han is currently a th year medical student at the Perdana University Royal College of Surgeons in Ireland School of Medicine, Perdana University, Kuala Lumpur, Malaysia

\section{Introduction}

Recent surveys have shown that placebo use rates vary internationally. In $2013,12 \%$ of general practitioners in the UK reported using a pure placebo at least once in their career, with $1 \%$ reporting use weekly. Almost all general practitioners (97\%) reported using an impure placebo at least once in their career ( $77 \%$ weekly).' Studies from Poland and the USA found that $79 \%$ and $45 \%$ of doctors had used placebo as a treatment, respectively. ${ }^{2,3}$

Despite its success as a medical intervention for some patients, ${ }^{4-8}$ the ethical controversy around placebo use continues.9-14 A 2012 study found that placebo use was not acceptable for two main reasons: it threatens the doctor-patient relationship and it deceives the patient. ${ }^{15}$ The lack of a clear definition of placebo adds to the confusion. ${ }^{1,16,17}$ Since Beecher's 1955 work "The Powerful Placebo", the effect has been considered a scientific fact, ${ }^{18}$ and our understanding of the term has evolved from considering only 'sugar pills' to acknowledging that placebo includes any condition that simulates a therapeutic environment. Placebo effects vary depending on not only whether or not a treatment is perceived to be administered, but how it is administered, and by whom. ${ }^{19}$ The potential benefit that might be realised if judicious and appropriate use of placebo were employed is large. The objective of this study was to explore doctors' understanding of the term placebo, mechanisms of action, its effectiveness, and concerns about use.

\section{Methods}

A questionnaire (in English and Malay) examining attitudes to and experiences of using placebo was developed based on existing questionnaires. ${ }^{15,16}$ Qualified hospital physicians working in a teaching hospital in Sarawak were recruited in person over a 3-month period (January-March 2015). Written consent was obtained from all respondents. Out of an estimated 360 doctors, 139 (38.6\%) from 12 departments, excluding palliative care, were invited to participate.

Survey questions included demographic items, items concerning the use of placebo, its effectiveness and opinions on use. Multiple responses were allowed for some items to capture as much information as possible. Respondents only responded to questions relevant to them. Respondents were also given a case in which a placebo was successful and asked to say why. Data was entered into SPSS for analysis. In the case of missing data for an item, the appropriate adjustments to the overall number of samples were made.

Ethical approval was granted by the Malaysian National Medical Research and Ethics Committee (NMRR-14-1155-21804(IIR)).

\section{Results}

A total of 76 doctors participated in the survey (response rate: $54.7 \%$ ). Mean age was 32.1 years (range: 24 to 52 ; standard deviation [SD]: 5.7$)$, and $38(52.1 \%)$ were female. Twelve (15.8\%) were junior doctors, $43(56.6 \%)$ were physicians, and $16(21.1 \%)$ were specialists. Average number of years of practice was 6.3 years (range: 0.1 to 27; SD: 5.4). Mean number of patients treated per week was 74.29 (range: 6 to 200, SD: 50.4). The main reason for non-participation in the study was the lack of time.

of the definitions given, "placebo is an intervention that is not expected to have an effect through a known physiological method"

Perdana University Royal College of Surgeons in Ireland School of Medicine, Perdana University, Serdang, Malaysia.

${ }^{2}$ International Medical University, Kuala Lumpur, Malaysia.

${ }^{3}$ Sarawak General Hospital, Kuching, Sarawak, Malaysia.

${ }^{4}$ Royal College of Surgeons in Ireland, Dublin, Ireland.

Correspondence:

Amanda Villiers Tuthill.

Address: Perdana University Royal College of Surgeons in Ireland School of Medicine, Perdana University, Jalan MAEPS Perdana, 43400 Serdang, Selangor, Malaysia.

Email: amanda.rhynehart@gmail.com 


\section{Short Communication}

was most endorsed (49.3\%), followed by "placebo is an intervention that is not considered to have any specific effect on the condition treated but with a possible unspecific effect" (38.4\%).

The reasons for placebo use, what was used, when and how are presented in Table 1. Most doctors, $(n=49,66.2 \%)$ never used pla- cebo, while $20.3 \%$ did so once a year on average and $2.7 \%$ daily. There were no significant differences in placebo use by gender, age or experience. of those who used placebo $(n=25), 71.9 \%$ used it for its possible psychological treatment effect and $28.1 \%$ for constantly complaining patients or to bridge a difficult treatment situation. Almost one third (31\%) used a placebo as a diagnostic tool.

Table 1. Placebo Use among Doctors at a Teaching Hospital in Malaysia

\begin{tabular}{|c|c|}
\hline Placebo Use & $\mathrm{n}(\%)$ \\
\hline \multicolumn{2}{|l|}{ What motivated you to prescribe or administer a placebo? } \\
\hline Because of a possible psychological treatment effect & $23(71.9)$ \\
\hline Because the patient was expecting a therapy & $8(25.0)$ \\
\hline To be able to still offer a treatment option to patients with untreatable, incurable disease & $5(15.6)$ \\
\hline Saline injection & $21(65.6)$ \\
\hline Vitamins & $16(50.0)$ \\
\hline Placebo pills & $6(18.8)$ \\
\hline Minerals and trace elements & $4(12.5)$ \\
\hline Herbal supplements & $2(6.3)$ \\
\hline Acupuncture & $1(3.1)$ \\
\hline Ibuprofen & $1(3.1)$ \\
\hline Sugar pills & $0(0.0)$ \\
\hline \multicolumn{2}{|l|}{ In which of the following situations have you prescribed or administered a placebo? } \\
\hline To calm a patient & $15(51.7)$ \\
\hline To mollify a complaining patient & $9(31.0)$ \\
\hline As a diagnostic tool & $9(31.0)$ \\
\hline It is a substance that may help and will not harm & $19(54.3)$ \\
\hline It is a medication & $13(37.1)$ \\
\hline It is a potentially beneficial medicine or treatment not typically used for their condition & $7(20.0)$ \\
\hline I said nothing & $6(17.1)$ \\
\hline It is a medicine with no specific effect & $4(11.4)$ \\
\hline It is a placebo & $3(8.6)$ \\
\hline This therapy has helped many other patients & $2(5.7)$ \\
\hline This is a highly effective drug or therapy & $1(2.9)$ \\
\hline This treatment promotes self-healing or the healing process & $1(2.9)$ \\
\hline I said what effect is expected from the therapy & $1(2.9)$ \\
\hline This is a treatment without pharmacological or physical effect for your condition & $0(0.0)$ \\
\hline \multicolumn{2}{|l|}{ When you use placebo, it is } \\
\hline Sometimes effective & $22(66.7)$ \\
\hline Usually effective & $7(21.2)$ \\
\hline I do not know if it is effective & $3(9.1)$ \\
\hline Never effective & $1(3.0)$ \\
\hline
\end{tabular}


Placebo users favoured saline injection (65.6\%) and vitamins $(50 \%)$. Most rejected the use of antibiotics $(68.6 \%)$ and ibuprofen $(57.1 \%)$ as placebos. Placebo use was most frequently thought to be acceptable if used for its psychological effect (74.3\%) or because it does not cause harm (34.3\%). The most common reasons for placebo use being unacceptable was the possibility of endangering the trust between doctor and patient $(59.2 \%)$ and deception of patient $(47.4 \%)$. Respondents thought that placebo use could be permitted if research $(59.5 \%$ ) or excellence in the department (24.3\%) supports its efficacy. More than $10 \%$ of respondents thought placebo use should be prohibited.

When prescribing placebo, $54.3 \%$ of the doctors informed the patient that "it is a substance that may help and will not harm", $37.1 \%$ informed patients that "it is a medication", and $17.1 \%$ said nothing. The majority $(87.9 \%)$ of prescribing doctors observed placebo was sometimes or usually effective. For the case, most doctors $(73.3 \%)$ thought that improvements in pain experienced were because the pain was psychogenic $(n=55,73.3 \%)$.

\section{Discussion}

The study provides information about doctors' perceptions of what a placebo is, its use, and attitudes towards placebo usage in a Malaysian context. Most respondents had not prescribed a placebo $(66.2 \%)$. Although this is consistent with a recent Canadian study $\left(23 \%\right.$ used placebo), ${ }^{20}$ it is inconsistent with other surveys reporting higher use. ${ }^{1,2}$ The reasons cited by doctors for non-use are consistent with other studies in which respondents were concerned about endangering trust or withholding information despite the prevalence of a paternalistic doctor-patient relationship in Malaysia. ${ }^{6}$ Perhaps the move towards evidence-based practice is significant as most doctors in this study felt that placebo use should only be permitted if supported by research. Most doctors reported prescribing placebo for possible psychological effects and in situations to calm patients. This may be because most doctors agreed that the mechanism of placebo is mostly psychological.

The placebo effect is influenced by a patient's positive expectation following a clinical encounter, ${ }^{21}$ as highlighted in open/hidden studies where a placebo effect is documented but no 'placebo' is used..$^{22}$ Over half of the placebo users in the current study informed their patients that the placebo was a substance 'that may help' while others referred to it as 'a medication'. Such language is likely to foster an expectation of beneficial effect in the patient. The power of expectation is very important in the therapeutic environment, yet the medical profession may underutilize this potentially effective tool as a result of poorly understanding it.

Almost a third of doctors reported using placebo as a diagnostic tool to distinguish between a genuine and imaginary symptom. It is acknowledged that use of placebos may result in an improvement in physical symptoms; hence the use of placebo in such a diagnostic capacity is, at best, ineffectual as physiological effects may occur. At worst it is dangerous as it may result in the non-treatment of genuine disorders and belies the effectiveness of placebo.

Most doctors surveyed thought the mechanism of action of placebo was psychological and felt that improvements in pain experienced as a result of placebo administration were because the pain had no organic cause and was psychogenic. These results highlight a lack of understanding of the physiological effects of placebo. While there appears to be an awareness of the power of expectation, when presented with a case of effective placebo treatment doctors appear somewhat reluctant to acknowledge the physiological power of placebo.

It has been suggested that difficulty in explaining to patients that a treatment may not help may reduce the potential placebo effect, leading doctors to use some form of deception such as not telling the patient directly that they are using a placebo. ${ }^{23,24}$ Respondents' perceptions regarding placebo effectiveness were ambivalent. While some doctors agreed that placebos are effective, most chose a neutral stance. There appears to be gaps in doctors' knowledge regarding placebos, how they work, and how they may be used, likely to limit the discussion of treatment options not specifically designed for the patient's complaint.

Limitations to this study included the small sample size and the limited setting of a single hospital (so results may not be generalizable to other hospitals in Malaysia). Small numbers of doctors were recruited from each department, hence the doctors were considered as a homogenous group and not representative of their individual departments. Further research is warranted into differing attitudes to placebo use among doctors specialising in areas such as pain management. The survey relied on doctors recalling how frequently they had used placebos in the past. Thus, we cannot determine the actual frequency of use. Strengths of this study include the use of a purpose designed questionnaire available in both English and Bahasa Malaysia. This study also contributes to the knowledge of attitudes to placebo use among healthcare professionals in Malaysia, an area which has been understudied to date.

The majority of doctors surveyed do not use placebos. A growing body of evidence on the usefulness of placebo raises the question of whether use should be recommended. This raises ethical challenges. Agreement on the range of interventions that are considered suitable to be used as placebo, and in what situations, would bring clarity to the discussion. A clear definition is needed to prevent conceptual confusion and further research should explore how doctors might harness the power of placebo without endangering the very relationship that may contribute to the effect.

\section{References}

1. Howick J, Bishop FL, Heneghan C, Wolstenholme J, Stevens S, Hobbs FD, et al. Placebo use in the United Kingdom: results from a national survey of primary care practitioners. PLOS One. 2013;8(3):e58247.

2. Babel P. Use of placebo interventions in primary care in Poland. Med Princ Pract. 2013;22(5):484-8.

3. Sherman R, Hickner J. Academic physicians use placebos in clinical practice and believe in the mind-body connection. J Gen Intern Med. 2008 Jan;23(1):710.

4. Kaptchuk TJ, Kelley JM, Conboy LA, Davis RB, Kerr CE, Jacobson EE, et al. Components of placebo effect: randomised controlled trial in patients with irritable bowel syndrome. BMJ. 2008 May 3;336(7651):999-1003.

5. Haour F. Mechanisms of the placebo effect and of conditioning. Neuroimmunomodulation. 2005;12(4):195-200.

6. Meissner K, Distel H, Mitzdorf U. Evidence for placebo effects on physical but not on biochemical outcome parameters: a review of clinical trials. BMC Med. 2007 Mar 19;5:3.

7. Zubieta JK, Bueller JA, Jackson LR, Scott DJ, Xu Y, Koeppe RA, et al. Placebo 


\section{Short Communication}

effects mediated by endogenous opioid activity on mu-opioid receptors. J Neurosci. 2005 Aug 24;25(34):7754-62.

8. Enck P, Benedetti $F$, Schedlowski M. New insights into the placebo and nocebo responses. Neuron. 2008 Jul 31;59(2):195-206.

9. Biller N. The placebo effect: mocking or mirroring medicine? Perspect Biol Med. 1999 Spring;42(3):398-401.

10. Biller-Andorno $\mathrm{N}$. The use of the placebo effect in clinical medicine-ethical blunder or ethical imperative? Sci Eng Ethics. 2004 Jan;10(1):43-50.

11. Bishop FL, Aizlewood L, Adams AE. When and why placebo-prescribing is acceptable and unacceptable: a focus group study of patients' views. PLOS One. 2014 Jul 9;9(7):e101822.

12. Kluge EH. Placebos: some ethical considerations. CMAJ. 1990 Feb $15 ; 142(4): 293-5$.

13. Simmons B. Problems in deceptive medical procedures: an ethical and legal analysis of the administration of placebos. J Med Ethics. 1978 Dec;4(4):172-81.

14. Thomas KB. The placebo in general practice. Lancet. 1994 Oct 15;344(8929):1066-7.

15. Meissner K, Höfner L, Fässler M, Linde K. Widespread use of pure and impure placebo interventions by GPs in Germany. Fam Pract. 2012 Feb;29(1):7985.

16. Fässler M, Gnädinger M, Rosemann T, Biller-Andorno N. Use of placebo interventions among Swiss primary care providers. BMC Health Serv Res. 2009 Aug 10;9:144.

17. Tilburt JC, Emanuel EJ, Kaptchuk TJ, Curlin FA, Miller FG. Prescribing “placebo treatments": results of national survey of US internists and rheumatologists. BMJ. 2008 Oct 23;337:a1938.

18. Kienle CS, Kiene H. The powerful placebo effect: fact or fiction? J Clin Epidemiol. 1997 Dec;50(12):1311-8.

19. Fässler M, Meissner K, Kleijnen J, Hróbjartsson A, Linde K. A systematic review found no consistent difference in effect between more and less intensive placebo interventions. J Clin Epidemiol. 2015 Apr;68(4):442-51.

20. Harris CS, Campbell NK, Raz A. Placebo trends across the border: US versus Canada. Plos One. 2015 Nov 25;10(11):e0142804.

21. Miller FG, Rosenstein DL. The nature and power of the placebo effect. J Clin Epidemiol. 2006 Apr;59(4):331-5.

22. Benedetti F, Carlino E, Pollo A. Hidden administration of drugs. Clin Pharmacol Ther. 2011 Nov;90(5):651-61.

23. Linde $\mathrm{K}$, Witt CM, Streng A, Weidenhammer W, Wagenpfeil S, Brinkhaus $B$, et al. The impact of patient expectations on outcomes in four randomized controlled trials of acupuncture in patients with chronic pain. Pain. 2007 Apr;128(3):264-71.

24. de Craen AJ, Kaptchuk TJ, Tijssen JG, Kleijnen J. Placebos and placebo effects in medicine: historical overview. J R Soc Med. 1999 0ct;92(10):511-5.

\section{Acknowledgments}

None.

Conflict of Interest Statement a Funding

The authors have no funding, financial relationships, or conflicts of interest to disclose.

\section{Author Contributions}

Conceptualization: KM. Data collection: TZH, CXC, AWC, CYW. Data analysis and interpretation, Writing: AVT, TZH, CXC, AWC, KM. Critical revision of the manuscript: AVT, KM. Approval of the final version: AVT, TZH, CXC, AWC, CYW, KM. Administrative/technical advice: CYW.

Cite as:

Tuthill AV, Han TZ, Chai CX, Chai AW, Wong CY, Morgan K. Attitudes to and understanding of placebo use: a cross-sectional exploratory study in a Malaysian hospital. 2016 Sep-Dec;4(3):108-11. 